\title{
Introduction: Conceptual Change and Its Models
}

\author{
Ismo T. Koponen
}

Published online: 12 March 2014

(C) Springer Science+Business Media Dordrecht 2014

This special issue of Science and Education contains selected articles from the Conceptual Change and Its Models (ConChaMo) workshops held in 2012-2013 in Helsinki, Department of Physics, University of Helsinki, made possible with the generous support from the Finnish Cultural Foundation. The workshops brought together researchers from diverse fields of science, cognitive science and educational sciences, in order to develop views and insight how conceptual learning and conceptual change takes place and could be facilitated in science learning.

Conceptual change is a central notion of many contemporary accounts of learning. It refers to learning process, where learners do not merely accumulate more knowledge, but where their conceptions of phenomena in a certain domain undergo a restructuring process, leading from common-sense beliefs to scientific conceptions. The conceptual change approach on learning has potential to transform science education, and bring it closer to the empirical and theoretical work on conceptual development, concept learning, and conceptual evolution in psychology, philosophy and cognitive science.

The general goal of the thematic special issue is to facilitate interdisciplinary communication so that progress will be made towards integrated theories of conceptual change. The aim is not only to bring researchers and ideas together, but also to unite them in the form of new types of research methods and theoretical models. Combining the approaches of the psychological, educational, philosophical, and computational sciences will result in deeper understanding of conceptual change.

Conceptual change, as a research paradigm itself, and how the different views on cognitive and psychological mechanisms have framed the conceptions of conceptual change, is discussed in the article Towards an Explanation for Conceptual Change: A Mechanistic Alternative by Anna-Mari Rusanen. She introduces some new viewpoint on understanding the conceptual change as a research paradigm, and argues that proper understanding of conceptual change hinges on proper description for the information processing task and on cognitive mechanisms responsible for those tasks.

I. T. Koponen $(\bowtie)$

Department of Physics, University of Helsinki, Helsinki, Finland

e-mail: ismo.koponen@helsinki.fi 
One well-known approach on conceptual change, known as framework theory, is discussed in article Conceptual Change from the Framework Theory Side of the Fence by Stella Vosniadou and Irini Skopeliti. They describe the framework theory approach to conceptual change explaining how children construct relatively coherent conceptual system, i.e. a framework theory of physical phenomena, based on everyday observations. On this basis, the authors discuss how many so called "misconceptions" are outcomes of mixing scientific information received though learning to the existing but incompatible naive physics frameworks. The framework theory and the theory of mind are used as the theoretical underpinnings in the article Using Theory of Mind to Promote Conceptual Change in Science by Natassa Kyriakopoulou and Stella Vosniadou, where young childrens' learning process is investigated. The authors present results of an empirical study, which exemplify how young children think about the phenomena in world and how they think about the difference between their beliefs and the beliefs of others.

A different viewpoint on students' misconceptions is provided in the article Considering Students' Conceptions as Complex Dynamic Systems by David E. Brown. The author challenges the views, which sees of students' naive ideas in science as unitary misconceptions. Instead, he proposes that we should see students' naive thoughts as a dynamic conceptual system, which is embedded in and embedding other dynamic systems. This opens up a new kind of thinking of conceptual change from the viewpoint of dynamic complex systems, with interesting and important theoretical and practical implications.

The empirical research on conceptual change has traditionally relied on classroom studies and teaching interventions. An interesting alternative on such approaches is suggested in article What can be learned from a laboratory model of conceptual change? Descriptive findings and methodological issues by Stellan Ohlsson and David Cosejo. These authors also note that researcher "have not converged on a single theory for conceptual change, nor has any one theory been decisively falsified"' and propose that laboratory models of conceptual change could be more illuminating. They introduce a recategorization experiment, which has the potential to give detailed information of the temporal dynamics of learning, revealing how initial conceptions are eventually transformed to the final ones.

In the article Confronting conceptual challenges in thermodynamics by use of selfgenerated analogies by Jesper Haglund and Fredrik Jeppsson, and it examines how students use self-generated analogies when they learn a new subject. The context of the study is thermodynamics, in the fourth year of pre-service teachers' studies. The results of the study show that students benefit from the self-generated analogies and they help students to get acquainted with the new subject.

The last article, Explanatory Identities and Conceptual Change by Paul Thagard discusses the identities of ordinary things with scientific ones. Thagard examines the history of 15 important identifications, among them e.g. air, earth, electricity, heat and light. As an outcome of this examination, new insights on the nature of justification, explanation, and conceptual change is gained.

In summary, this special issue give a glimpse of different approaches on the concept learning and conceptual change, and show how interdisciplinary approaches grounded in education, cognitive science and learning psychology can help us to form a better and more comprehensive picture of the problems in learning and instruction, and how on this knowledge, new ideas and suggestions for better learning emerge. 